\title{
THE ENGINE MECHANICAL COORDINATES MEASURING IN THE ASYNCHRONOUS MOTOR
}

\author{
Yuriy N. Dementyev, Anara D. Umurzakova \\ National Research Tomsk Polytechnic University, 634050 Tomsk, Russia
}

\begin{abstract}
The article considers indirect control devices for the mechanical coordinate's measurement of a three-phase asynchronous electric motor, which detects the temperature of the stator winding conductors and the basic frequency of stator voltage. Characteristics of mechanical coordinate's simulation of the asynchronous motor are provided to assess the accuracy of the mechanical coordinate's measurement in the dynamic modes.
\end{abstract}

\section{Introduction}

At present, asynchronous motors form the basis of an electric drive of most mechanisms used in all industries. Due to the simplicity, reliability in operation and reduced cost compared to other types of engines asynchronous motors are widely used in industry. They drive the final elements of general-purpose mechanisms for various purposes. The simplest in design and the most reliable in operation are squirrel-cage asynchronous motors.

In the analysis of modern electric motors of general industrial machinery and systems used in the industry in the technological processes, there is often the need to control the mechanical coordinates, namely, of the angular velocity and torque of squirrel-cage asynchronous motors.

The most common devices of mechanical coordinates monitoring of an asynchronous electric drive are the measuring devices velocity [3, 5, 9] and torque sensors [3, 5, 8] implemented on the basis of additional DC or AC micromachines built into the electric motor or attached to it. But these devices are expensive and have a complicated design; the thorough mechanical connection to the rotating parts of the motor is required, in some cases, the installation of a velocity sensor on the shaft is simply impossible. Therefore, it is actual to use other means of mechanical coordinates monitoring of general industrial devices, such as indirect control devices, when they are applied, these disadvantages are eliminated $[1,2,4,6,7]$.

\section{Problem statement}

In this regard, the objective of the research is to consider the options for devices of indirect control of coordinates - the angular velocity of rotation and torque of a three-phase squirrel-cage asynchronous motor. Moreover, unlike the existing devices, in the proposed ones the control is simplified, and there is a possibility of accounting for temperature of conductors of a stator winding, the frequency of a fundamental harmonic of the stator voltage, temperature coefficient, which depends on the conductor material, that improves the accuracy of the coordinates of the three-phase asynchronous motor.

\section{Mathematical model}

Mathematical model to the angular velocity of rotation $\omega(t)$ of a three-phase squirrel-cage motor describes the equation

$$
\omega(t)=\omega_{m e s}(t) \cdot\left[1+\Delta \omega_{\mathrm{int}}(t)+\Delta \omega_{d i f}(t)\right],
$$

where

$$
\begin{aligned}
& \omega_{\text {mes }}(t)=\frac{\sqrt{3} \cdot\left[u_{a}(t)-\left(z+R_{r \alpha}^{\prime}\right) \cdot i_{a}(t)\right]}{\int_{0}^{1 / f}\left(z \cdot\left[i_{a}(t)+2 \cdot i_{b}(t)\right]-\left[u_{a}(t)+2 \cdot u_{b}(t)\right]\right) d t-L_{\beta} \cdot\left[i_{a}(t)+i_{b}(t)\right]}-\text { the measured value of the angular velocity; } \\
& \Delta \omega_{\text {int }}(t)=\frac{\int_{0}^{1 / f}\left[u_{a}(t)-z \cdot i_{a}(t)\right] d t}{T_{r}^{\prime} \cdot\left[u_{a}(t)-\left(z+R_{r \alpha}^{\prime}\right) \cdot i_{a}(t)\right]}-\text { the dynamic integral component of the relative value of the angular velocity; }
\end{aligned}
$$


$\Delta \omega_{\text {dif }}(t)=\frac{L_{\beta} \frac{d i_{a}(t)}{d t}}{u_{a}(t)-\left(z+R_{r \alpha}^{\prime}\right) \cdot i_{a}(t)}$ the dynamic differential component of the relative value of the angular velocity;

$i_{a}, i_{b}, u_{a}, u_{b}$ - currents and voltage of correspondently phases A and $\mathrm{B}$ of the stator winding;

$f$ - the frequency of a fundamental harmonic of the voltage supply of an asynchronous motor;

$R_{r \alpha}^{\prime}=R_{r}^{\prime} \cdot \alpha$ - active reduced resistance of stator winding taking into account $\alpha$;

where $\quad R_{r}^{\prime}$-active reduced resistance of a stator;

$\alpha=\frac{L_{S}}{L_{r}^{\prime}}$ - the coefficient equals the ratio of total inductance of the stator winding $L_{s}$ to reduced total inductance of the stator winding $L_{r}^{\prime}$;

$L_{\beta}=L_{\mu} \cdot \beta-L_{s}$ - the inductance taking into account the coefficient $\beta$;

where $\quad L_{\mu}-$ mutual inductance of stator and rotor windings;

$\beta=\frac{L_{\mu}}{L_{r}^{\prime}}-$ the coefficient is equal to the ratio of mutual inductance $L_{\mu}$ to the reduced total inductance of the rotor winding $L_{r}^{\prime}$

$T_{r}^{\prime}=\frac{L_{r}^{\prime}}{R_{r}^{\prime}}$ - the rotor time constant.

Active resistance of the stator winding taking into account the temperature coefficient $\alpha_{t}$ is determined by the following expression:

$$
z=R_{s} \cdot\left[1+\alpha_{t} \cdot\left(t_{c o n}-20\right)\right]
$$

where $\quad R_{s}$ - active resistance of the stator winding;

$t_{c o n}$ - the temperature of conductors of the stator winding of the asynchronous motor.

Mathematical model to the value of the torque of a three-phase squirrel-cage asynchronous motor describes the equation

$$
M(t)=\sqrt{3} \cdot p_{n} \cdot\left[i_{a}(t) \cdot \int_{0}^{1 / f}\left[u_{b}(t)-z \cdot i_{b}(t)\right] d t-i_{b}(t) \cdot \int_{0}^{1 / f}\left[u_{a}(t)-z \cdot i_{a}(t)\right] d t\right],
$$

where $p_{n}-$ the number of pairs of motor poles.

\section{Experimental setup and methods}

Figure1 shows the scheme of the angular velocity change of rotation of a three-phase squirrel-cage asynchronous motor.

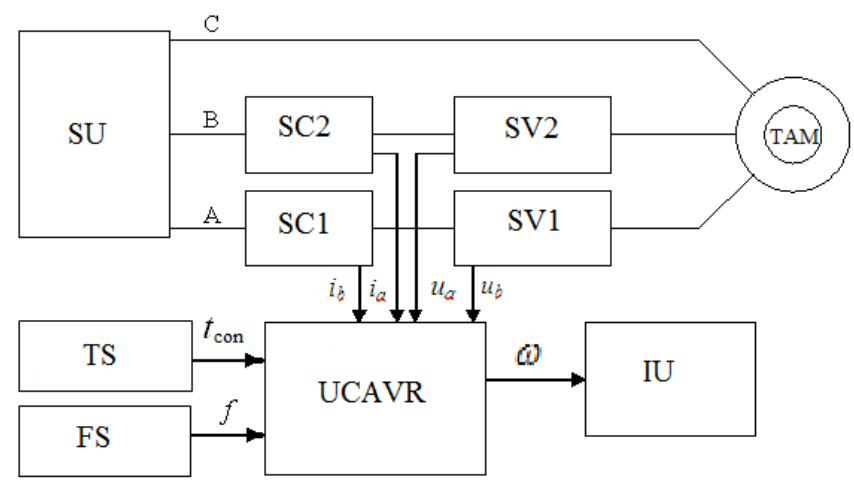

Figure1. The scheme of the angular velocity change of rotation of a three-phase squirrel-cage asynchronous motor.

The scheme includes the following units: a unit of calculating the angular velocity of rotation (UCAVR), an indexing unit (IU); power supply unit (SU); current sensors (CS1 и CS2), voltage sensors (VS1 и VS2), temperature sensors (TS), a frequency sensor (FS ) and a three-phase asynchronous motor (TAM).

As for voltage sensors (VS1 и VS2) voltage transformers are used, for current sensors (CS1 и CS2) - current transformers, a temperature sensor is a posistor, (TS ) - a frequency sensor. UCAVR (fig. 2) is implemented by the standard equipment on the basis of integrated circuits or microprocessor sets.

The unit of calculating the angular velocity of rotation (UCAVR) constitutes:

SU1 - a summing unit;

UCDDC - a unit of calculating the dynamic differential component of the relative value of the angular velocity; 
UCDIC - a unit of calculating the dynamic integral component of the relative value of the angular velocity;

CEU1 - a coefficient entry unit;

MU1 - a multiplier unit;

UCMV - a unit of calculating the measured value of the angular velocity.

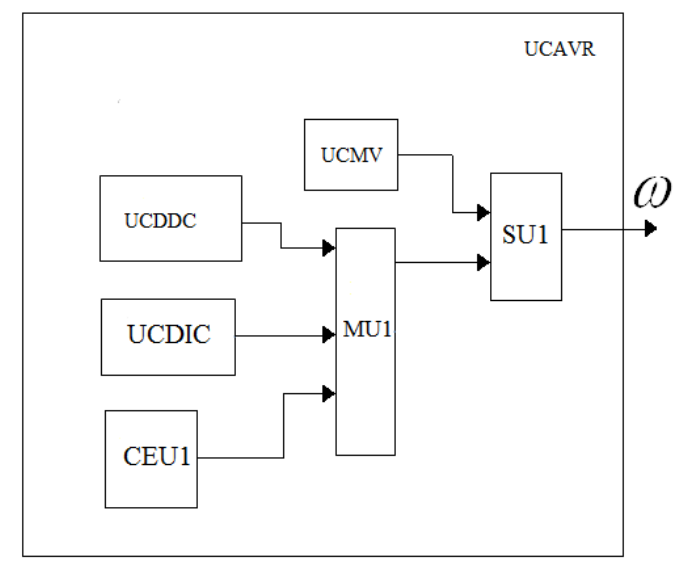

Figure 2. The unit of a squirrel-cage asynchronous motor.

At the output of calculating the angular velocity of rotation of a three-phase asynchronous motor with squirrel-cage rotor (UCAVR) is formed by the value of the angular velocity of rotation.

By means of the scheme of fig. 3 it is possible to measure the torque of the squirrel-cage asynchronous motor.

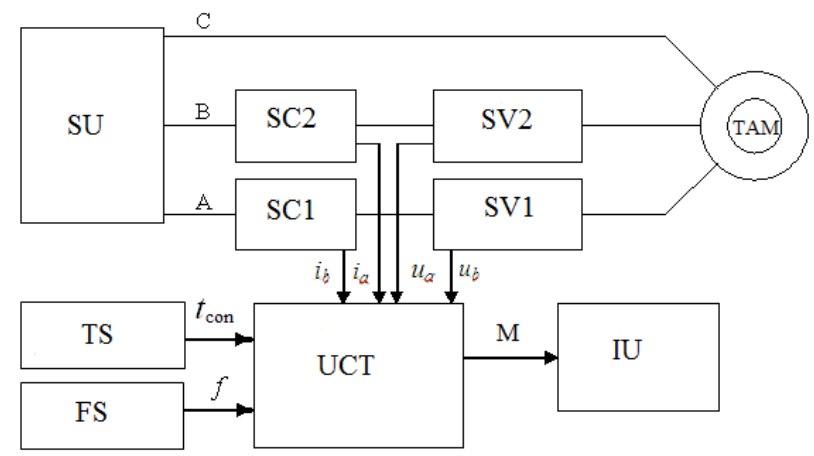

Figure 3. The scheme for measuring the torque of the squirrel-cage asynchronous motor.

The components that are essential for the implementation of this scheme are similar to Fig. 1.

The main difference is the element to calculate the torque (UCT) (Figure 4), which consists of units of calculating the resistance of the stator windings taking into account the temperature coefficient (UCRST), multiplication units (MU1, MU2, MU3, MU4, MU5), adding units (AU1, AU 2, AU 3), integration units (IU1, IU2), coefficients input units(CIU1, CIU 2), period calculation unit (PCU).

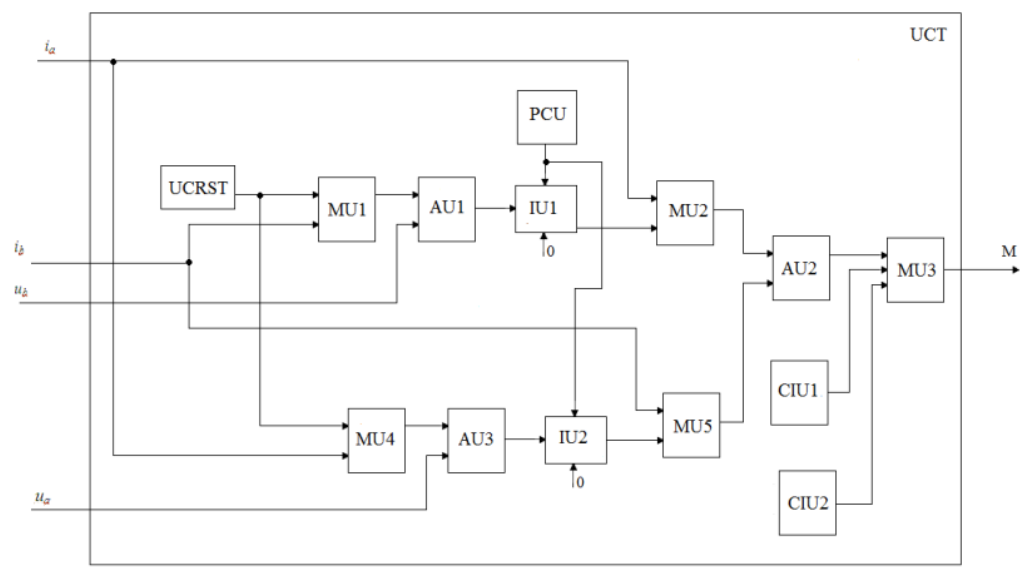

Figure 4. The unit of calculating the torque of the squirrel-cage asynchronous motor.

At the output the unit of calculating the torque (UCT) generates the value of a signal proportional to the value of the torque of a three-phase squirrel-cage asynchronous motor. 


\section{Results and discussion}

To determine the angular velocity of rotation and torque of a squirrel-cage asynchronous motor a simulation model of a mechanical coordinates measuring device in the software package Matlab has been created. Moreover, the studies have been carried out for the devices discussed in this article, as well as devices [1,2], which do not take into account the effect of temperature of the stator winding conductors and fundamental frequency of the stator voltage.

In the simulation modeling, zero initial conditions have been adopted, modeling start time $t_{0}=0$; modeling finish time $t_{\mathrm{K}}=50$ $\mathrm{s}$; integration step $\Delta \mathrm{t}=0,001 \mathrm{~s}$ and the run mode of a squirrel-cage asynchronous motor as the heaviest one, in which the parameters vary widely, has been studied.

Figures 5 and 6 show the characteristics of the angular velocity of rotation $\omega(t)$ and the torque $M(t)$ where curvel is the characteristic obtained by using the device $[1,2]$, and curve 2 is obtained using the proposed devices.
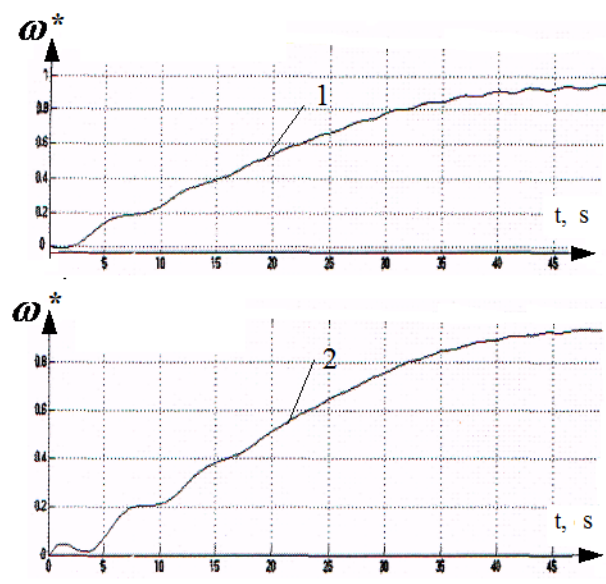

Figure 5. The characteristics of the angular velocity of rotation $\omega(t)$ of a squirrel-cage asynchronous motor.

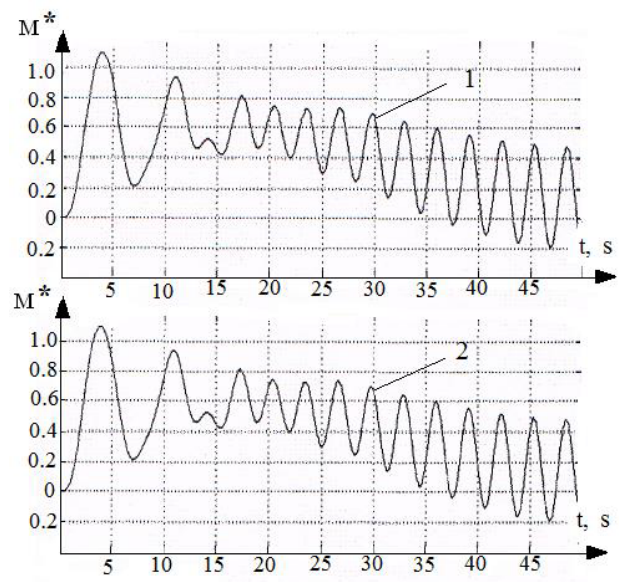

Figure 6. The characteristics of the torque $M(t)$ of a squirrel-cage asynchronous motor.

The comparison of characteristics of the angular velocity of rotation $\omega(t)$ and the torque $M(t)$ obtained by using measuring devices $[1,2]$, with the characteristics of the angular velocity and the torque of rotation obtained by the proposed device indicates that the accuracy of measurement in the first case is $94-95 \%$, and in second - 96-97\%.

The table 1 shows the results of the simulation modeling for a squirrel-cage asynchronous motor $4 \mathrm{~A} 50 \mathrm{~A} 4 \mathrm{P}_{2}=60 \mathrm{~W}, \mathrm{U}_{\text {н. }}=220$ $\mathrm{V}, 2 \mathrm{p}=4, \mathrm{f}=50 \mathrm{~Hz}$.

Table 1. The results of the simulation modeling for a squirrel-cage asynchronous motor 4A50A4

\begin{tabular}{|l|c|c|}
\hline \multicolumn{1}{|c|}{ Title } & $\omega_{\max }, \mathrm{rad} / \mathrm{s}$ & $M_{\max }, \mathrm{N} \cdot \mathrm{M}$ \\
\hline The direct measurement of the coordinates & 156,8 & 0,812 \\
\hline $\begin{array}{l}\text { Measuring, using the device of indirect control of mechanical coordinates } \\
\text { without taking into account the temperature conductors of the stator } \\
\text { winding, the fundamental frequency of the stator voltage }\end{array}$ & 148,96 & 0,763 \\
\hline $\begin{array}{l}\text { Measuring, using the device of indirect control of mechanical coordinates } \\
\text { taking into account the temperature conductors of the stator winding, the } \\
\text { fundamental frequency of the stator voltage. }\end{array}$ & 151,3 & 0,772 \\
\hline
\end{tabular}




\section{Conclusions}

The conducted research proves that the devices proposed in the paper have the improved accuracy of measurement of mechanical coordinates of a squirrel-cage asynchronous motor in the dynamic working modes of an electric drive and a simpler implementation.

This research work has been completed within the state project «Science» 2.1318.2014.

\section{References}

1. Innovative Patent № 20031 Bulletin №2, 14.02.2009 Method of measuring the torque of the asynchronous motor / V.Yu. Melnikov, A.D. Umurzakova, Russ (2009)

2. Leytman M.B. Automatic measurement of the output parameters of electric motors, Russ. (1983)

3. V.Yu. Melnikov, A.D. Umurzakova Methods for measuring the angular velocity of rotation and torque of the asynchronous motor // Energy: Environment, safety, security: materials of XVI All-Russian scientific-technical conference, Tomsk: Publishing House, Russ (2010)

4. Potapov L.A. Measuring torques and rotational speed of micromotors, Russ. (1976)

5. Preliminary Patent of RK 18934, Bulletin № 11, 15.11.2007. The method for measuring torque of the asynchronous motor / Melnikov V.Yu., Umurzakova A.D., Russ. (2007)

6. Preliminary Patent of RK № 18973, Bulletin № 8, 15.08.2008. The method for measuring the angular velocity of rotation of a three-phase asynchronous motor / Melnikov V.Yu., Umurzakova A.D., Russ. (2008)

7. Rybalchenko Yu.I. Magnetoelastic torque sensors, Russ. (1981)

8. Tun A.Ya. The system of speed control of the electric drive, Russ. (1984) 